\title{
O impacto da COVID-19 na saúde mental do enfermeiro atuante na pandemia: uma revisão bibliográfica
}

The impact of COVID-19 on the mental health of nurses working in the pandemic: a literature review

El impacto del COVID-19 en la salud mental de las enfermeras que trabajan en la pandemia: una revisión de la literatura

Bianca Miranda Montelo

ORCID: https://orcid.org/0000-0002-2336-1314 Faculdade Master de Parauapebas, Brasil E-mail: biancamontelo@gmail.com

Edivaldo do Nascimento Sindeaux ORCID: https://orcid.org/0000-0001-8294-3253 Faculdade Master de Parauapebas, Brasil E-mail: edysindeaux@gmail.com

Stefane Carvalho da Cunha

ORCID: https://orcid.org/0000-0001-9426-5242 Faculdade Master de Parauapebas, Brasil E-mail: stefane.carvalhoo@hotmail.com

Vanessa Christielle Silva de Sousa ORCID: https://orcid.org/0000-0002-3025-439X Faculdade Master de Parauapebas, Brasil E-mail: vanessachrissousa@gmail.com

Victória Carolline do Moraes Gatti ORCID: https://orcid.org/0000-0002-7400-1685 Universidade Federal Rural da Amazônia, Brasil E-mail: victoriagatti.agro@gmail.com

Henrique da Silva Barata

ORCID: https://orcid.org/0000-0001-6356-4629 Universidade Federal Rural da Amazônia, Brasil E-mail: henriquebarata2000@gmail.com

Claudete Rosa da Silva ORCID: https://orcid.org/0000-0001-5063-8932 Universidade Federal Rural da Amazônia, Brasil E-mail: claudete.silva@ufra.edu.br

Priscilla Andrade Silva ORCID: https://orcid.org/0000-0002-2774-3192 Universidade Federal Rural da Amazônia, Brasil E-mail: priscilla.andrade@ufra.edu.br

Aliny Lopes Coelho

ORCID: https://orcid.org/0000-0001-9139-8832 Faculdade Master de Parauapebas, Brasil E-mail: alinylopesenf@gmail.com

\begin{abstract}
Resumo
O objetivo desse estudo foi identificar os principais impactos psicológicos vivenciados pelos profissionais de enfermagem atuante na linha de frente da pandemia da COVID-19, relatar os sintomas mais abrangentes e por quê geram o estresse e descrever as estratégias de coping para melhor enfrentamento da pandemia e evitar ao adoecimento psíquico. Realizou-se uma revisão sistemática da literatura, fundamentada nos agravos causados à saúde do trabalhador em tempos de pandemia. Recursos humanos insuficiente, altas jornadas de trabalho, medo de contágio e contágio a outras pessoas, a falta de membros na equipe, precariedade na descrição dos protocolos, a carência de formação profissional adequada, incerteza das medidas medicamentosas para controle da doença, condições insalubres dos serviços de saúde que podem vir a ocasionar adoecimento. Destas, os mais citados foram o estresse, a ansiedade, depressão, insônia, medo e estresse pós-traumático, O estresse ocupacional, Síndrome de Burnout, esgotamento emocional, angústia e distúrbios do sono. $\mathrm{O}$ apoio psicológico e melhores condições de trabalhos são essenciais para que o profissional de enfermagem exerça o cuidado de qualidade de forma contínua. Frente a essa situação, se faz necessário implantar medidas de prevenção para minimizar os impactos na saúde mental do
\end{abstract}


enfermeiro atuante na pandemia COVID-19 que são as ações psicológicas direcionadas aos profissionais da enfermagem, implantação de treinamentos adequados por meio das instituições de saúde bem como, incentivo a práticas integrativas como meditação, Yoga, e atividades similares para a redução do estresse, ter o descanso adequado entre as jornadas de trabalho junto a uma boa alimentação.

Palavras-chave: COVID-19; Impactos psicológicos; Estratégias de coping; Boa alimentação.

\begin{abstract}
The aim of this study was to identify the main psychological impacts experienced by nursing professionals working on the front line of the COVID-19 pandemic, to report the most comprehensive symptoms and why they generate stress and describe coping strategies to better cope with the pandemic and avoid psychic illness. This is a systematic review of the literature. It is based on the injuries caused to the health of the worker in times of pandemic. The review showed how resulted, that human recourses are insufficient with high working hours, providing fear of being contaminated and contagion to other people, lack of team members, precariousness in the description of protocols, lack of adequate professional training, uncertainty of drug measures to control the disease, conditions unhealthy health services that may cause illness. Of these, the most cited were stress, anxiety, depression, insomnia, fear and posttraumatic stress, occupational stress, Burnout Syndrome, emotional exhaustion, anguish, and sleep disorders. We found that psychological support and better working conditions are essential for the nursing professional to exercise quality care continuously. In view of this situation, it is necessary to implement prevention measures to minimize the impacts on mental health of nurses active in the COVID-19 pandemic, which are: implantation adequate training through health institutions and encouraging integrative practices such as meditation, yoga, and similar activities to reduce stress, having adequate rest between working days with a good power supply.
\end{abstract}

Keywords: COVID-19; Psychological impacts; Coping strategies; Good nutrition.

\title{
Resumen
}

El objetivo de este estudio fue identificar los principales impactos psicológicos experimentados por los profesionales de enfermería que trabajan en la primera línea de la pandemia COVID-19, reportar los síntomas más amplios y por qué generan estrés, y describir estrategias de afrontamiento para afrontar mejor la pandemia y evitar enfermedades mentales. enfermedad. Se trata de una revisión bibliográfica sistemática. Se basa en el daño causado a la salud del trabajador en tiempos de pandemia. La revisión arrojó, que los recursos humanos son insuficientes, con largas jornadas laborales, provocando temor de contaminación y contagio a otras personas, la falta de integrantes del equipo, la precariedad en la descripción de los protocolos, la falta de formación profesional adecuada, la incertidumbre de Medidas farmacológicas para el control de la enfermedad, condiciones insalubres de los servicios de salud que pueden provocar enfermedades. De estos, los más citados fueron el estrés, la ansiedad, la depresión, el insomnio, el miedo y el estrés postraumático, el estrés laboral, el Síndrome de Burnout, el agotamiento emocional, la angustia y los trastornos del sueño. Encontramos que el apoyo psicológico y mejores condiciones laborales son fundamentales para que el profesional de enfermería brinde una atención de calidad de manera continua. Ante esta situación, es necesario implementar medidas preventivas para minimizar los impactos en la salud mental de los enfermeros que laboran en la pandemia COVID-19, que son: implementación de capacitación adecuada a través de instituciones de salud y fomento de prácticas integradoras como la meditación, el yoga, y actividades similares para reducir el estrés, con un descanso adecuado entre los días laborales y una buena alimentación.

Palabras clave: COVID-19; Impactos psicológicos; Estrategias de afrontamiento; Buena alimentacion.

\section{Introdução}

Por ser uma atividade social, o trabalho exerce um papel fundamental nas condições de vida do ser humano. Gera efeito positivo, quando é capaz de satisfazer as necessidades básicas de subsistência. Todavia, ao realizá-lo, o homem expõe-se constantemente aos riscos presentes no ambiente laboral, os quais podem afetar diretamente em sua condição de saúde (Areosa, 2021).

O cotidiano desenvolvido pelo profissional de enfermagem gera uma dualidade de sentimentos - como, o prazer e o sofrimento -, devido a possibilidade de ser útil enquanto servem, ajudam e confortam, mas, ao se deparar com o sofrimento alheio, a dor, a morte e situações difíceis de serem superadas, o trabalhador sofre (Humerez, Ohl \& Silva, 2020).

Os fatores estressores relacionados à atuação da enfermagem tendem a se exacerbarem diante de um cenário de calamidade como o qual têm se instalado nos últimos meses, pois o mundo atualmente está passando por um período de turbulência decorrente da pandemia ocasionada pelo novo coronavírus. (Dal' Bosco et al., 2020).

O novo agente do coronavírus foi descoberto em 31 de dezembro de 2019, após casos registrados em Wuhan, na 
China. Uma doença de fácil transmissão entre as pessoas, sejam elas assintomáticas ou não, ressalta o estudo de Pereira et al. (2020).

A Organização Mundial da Saúde (OMS) declarou, em 30 de janeiro de 2020, que o surto da doença tornou emergência de Saúde Pública de importância internacional. A pandemia foi declarada em 11 de março de 2020 pela OMS pela sua rápida expansão no mundo, e desde então, causa impactos na população e prejuízos irreparáveis na economia, saúde pública e afeta o cotidiano (Ramos-Toescher et al., 2020).

O ambiente de trabalho é permeado por fatores que interferem, negativamente, na saúde do trabalhador. Tal interferência pode comprometer a sua realização como profissional, bem como a sua capacidade para desenvolver as atividades laborais, o que pode repercutir na qualidade da assistência prestada. Apesar do avanço do conhecimento acerca da saúde do profissional de enfermagem, persistem situações que podem predispor ao adoecimento laboral. (Da Luz et al., 2020)

Em consideração aos dados apresentados e o contexto da pandemia, esse estudo tem como objetivo identificar os principais impactos psicológicos causados pela pandemia da COVID-19 nos profissionais de enfermagem; relatar os sintomas mais abrangentes e por quê geram o estresse e descrever as estratégias de coping, (mecanismo para enfrentamentos), para evitar ao adoecimento psíquico.

\section{Metodologia}

Esta pesquisa utilizou como método a revisão integrativa da literatura. Segundo Souza, Silva e Carvalho (2010), "é um método que proporciona a síntese de conhecimento e a incorporação da aplicabilidade de resultados de estudos significativos na prática". Por se tratar de um estudo de revisão sistemática da literatura, o objetivo deste não envolve seres humanos, logo, não há a necessidade de aprovação por Comitê de Ética em Pesquisa (CEP) para a pesquisa. A opção por esta modalidade se dá por incluir diferentes tipos de estudos, que permite uma visão panorâmica do estudo de interesse.

O estudo em questão compreendeu as etapas a seguir: (1) Identificação do tema e objetivos; (2) Definição do método e estabelecimento dos critérios de seleção; (3) Seleção dos artigos na base; (4) Categorização dos estudos e definição das informações que irão ser extraídas; (5) Avaliação dos estudos selecionados para a revisão integrativa (6) Interpretação dos resultados (7) Discussão da revisão (Figura 1).

Para responder ao objetivo proposto, foram utilizadas as palavras chaves: enfermagem, COVID-19 e saúde mental. Definiu-se como critério de inclusão: artigos publicados nos anos de 2020 e 2021; publicados nacionalmente, em português, com as seguintes palavras chaves "enfermagem", "COVID-19", "saúde mental", que foram avaliadas e analisadas conforme o tema em questão. Foram excluídos artigos que envolvem outros profissionais da área da saúde, artigos que não foram encontrados em fontes confiáveis, dissertações, teses, livros, capítulos, relatos de experiência, estudos onde focam somente nas vivencias do paciente e que não se enquadram ao tema proposto. 
Figura 1 - Processo de identificação e inclusão dos estudos nas bases de dados Periódico CAPES, LILACS, Scielo e Biblioteca Virtual e Saúde (BVS).

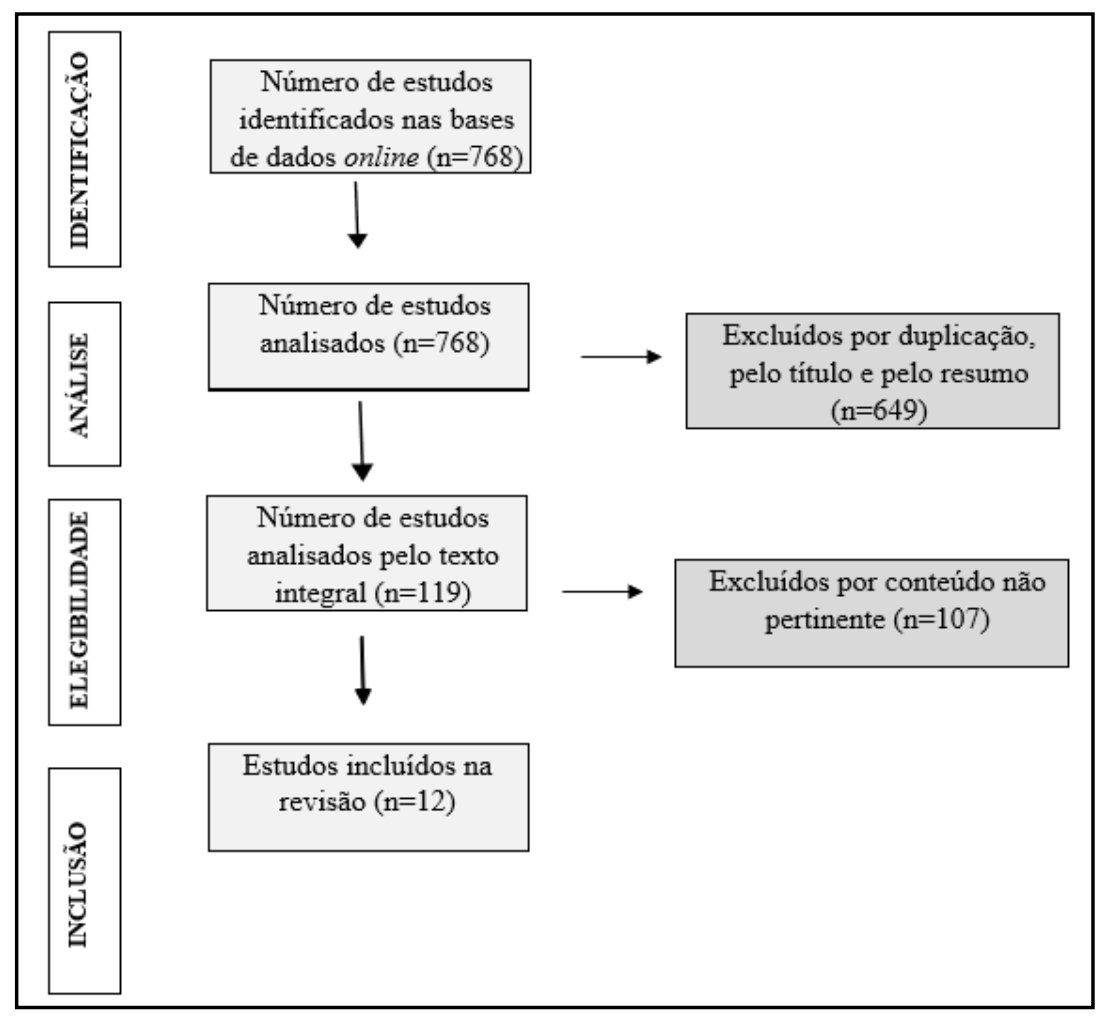

Fonte: Autores (2021).

O processo de busca ocorreu no período de março a junho de 2021, nas bases de dados Periódico CAPES, LILACS, Scielo e BVS (Biblioteca Virtual em Saúde). As dúvidas foram discutidas posteriormente até estabelecer um consenso dos autores. A análise dos estudos foi realizada de forma descritiva, onde possibilitou observar, descrever e classificar os dados sobre o impacto que a pandemia da COVID-19 causou na saúde mental do enfermeiro, que foi exposto e analisado através da Figura 1. Não houve a utilização de ferramentas digitais para categorização e análise dos resultados.

\section{Resultados e Discussão}

Foram identificados, nas bases de dados selecionadas, o total de 768 artigos de potencial relevantes; 649 foram eliminados por duplicação, o que restou apenas 119 para leitura e análise integral. O estudo resultou em doze trabalhos selecionados, que atenderam aos pré-requisitos dos critérios de inclusão e exclusão, que são estudos publicados entre 2020 e 2021 (Quadro 1). 
Quadro 1 - Artigos do corpus - por autoria e ano de publicação, título do artigo, metodologia do estudo, objetivos do estudo, e principais resultados.

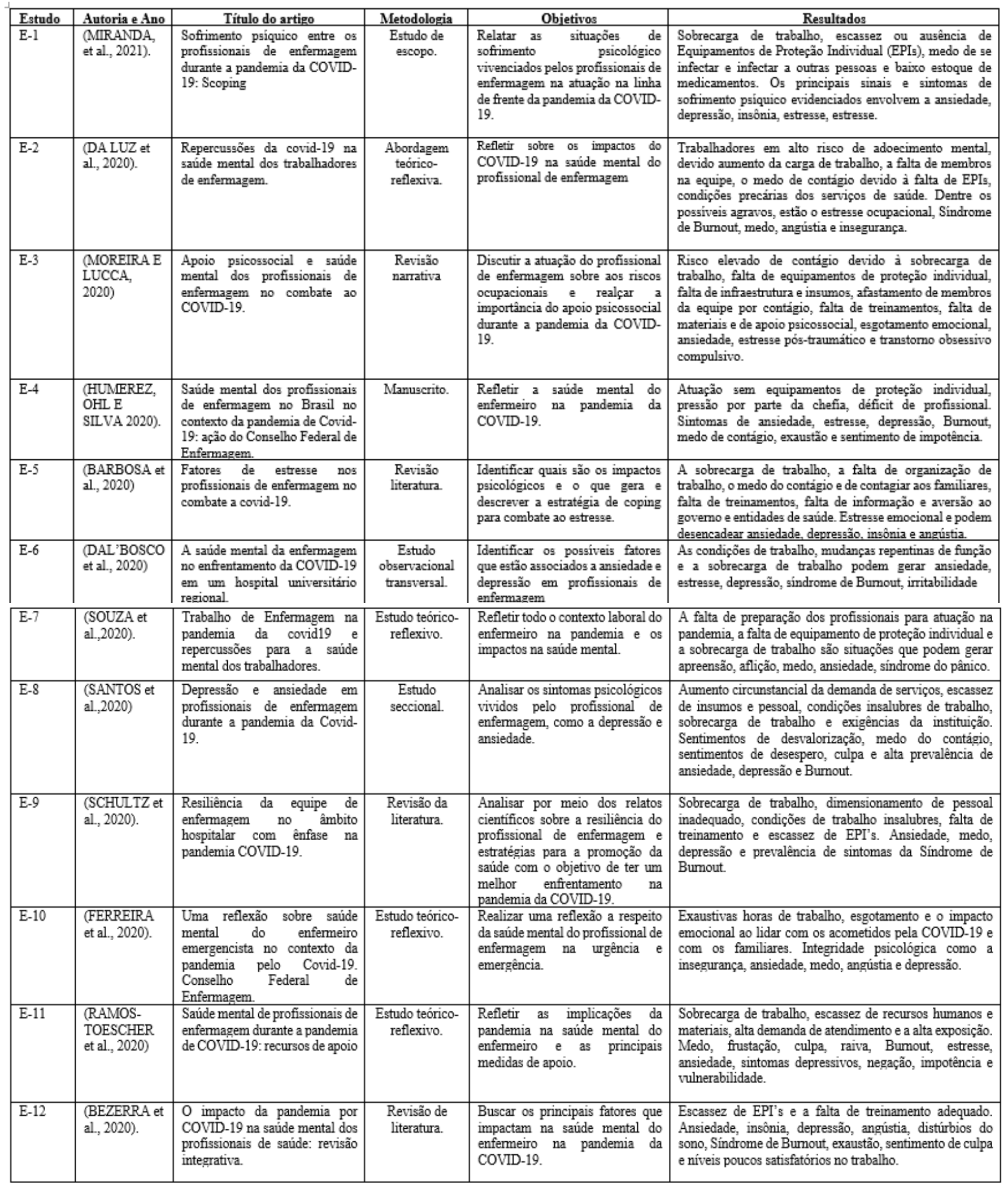

Fonte: Autores (2021).

Os doze artigos selecionados, chamados de E-1 ao E-12 (E = estudo), compuseram o corpus deste estudo, com as informaç̃̃es dos mesmos em categorias e estão apresentadas da seguinte forma: ano de publicação e autoria; título do artigo; 
metodologia do estudo; principais resultados, disponibilizados nos Quadro 1.

Foram identificados como os principais fatores laborais sendo a sobrecarga de trabalho, escassez de EPI's, condições laborais insalubres, a falta de organização de trabalho incluindo o déficit de membros da equipe e a ausência de treinamentos inadequados. Esses fatores são possíveis desencadeadores de sintomas de sofrimento psíquico, como constam nos estudos, que se se deve não só a natureza da atividade que desenvolvem; que está diretamente relacionada a sofrimentos emocionais daqueles a quem estes prestam seus serviços, mas também as condições de trabalho e falta de reconhecimento profissional. (Santos et al., 2020).

Miranda et al. (2020) evidenciaram que os profissionais de enfermagem enfrentam impactos psicológicos desencadeados pelas condições de trabalho, onde manifestam sintomas como depressão, ansiedade e estresse por um tempo significativo, também ressaltados pelos estudos do E-2 ao E-12.

O atual surto da pandemia da COVID-19 intensificou o estresse nos profissionais de enfermagem. Exercer a profissão exige lidar com a dor, sofrimento, mortes junto as condições precárias e a remuneração que não condiz com o esperado. É o que ressaltam Humerez, Ohl e Silva (2020) ao afirmar que "o exercício do profissional da enfermagem é marcado por múltiplas exigências: lidar com dor, sofrimento, mortes e perdas, a que se somam as condições desfavoráveis de trabalho e baixa remuneração."

A sobrecarga na jornada de trabalho do profissional da enfermagem pode ser um dos principais fatores apontados como causador do estresse e da ansiedade e pode ocasionar acidentes no trabalho e erros de medicação devido à exaustão e ao esgotamento profissional. Afirmam Barbosa et al. (2020) que o trabalho em excesso pode contribuir para os problemas relacionados a saúde mental e física dos profissionais da área da enfermagem, bem como, acidentes no trabalho e erros de medicação e dessa forma contribui para o estresse emocional.

Concomitante a esse impacto, a alta jornada de trabalho é capaz de gerar sentimento de impotência e sofrimento em função da necessidade de priorização das atividades, ou seja, vem o sentimento de não conseguir atender a todas as demandas do paciente. Com base nos estudos de Humerez, Ohl e Silva (2020), cuidar da vida em sofrimento e morte pode acarretar sentimento de impotência e pode desencadear sofrimento proveniente da frustração no trabalho.

Uma das medidas adotadas para minimizar o contágio da pandemia da COVID-19 tem sido o isolamento social, que reflete na perda de apoio social (Santos et al.,2020) salienta que ao considerar a natureza sociável dos indivíduos, que possuem necessidades de interações e que tais interações são fundamentais para construção individual, desenvolvimento, aprendizagem, ensino, criação de vínculos; o distanciamento tem potencial para ser um fator negativo na reestruturação psíquica.

Outro fator que pode contribuir para causar o estresse, ansiedade e depressão é a falta de Equipamentos de Proteção Individual (EPI's) que pode colocar o profissional de enfermagem em grave risco de contaminação, aumentar o número de contágio devido à exposição e ocasionar a redução de profissionais da enfermagem. Essa redução tem potencial para ocasionar uma crise na saúde pública. Moreira e Lucca (2020) enfatizam que a falta de recursos materiais, como EPI's e equipamentos necessários para realizar um cuidado de qualidade ao paciente e déficit de membros da equipe, o que contribui para a sobrecarga física e psíquica ao profissional de enfermagem que atua na pandemia.

Muitos remanejamentos de funções ocorreram nesse período de pandemia, como aponta o estudo E-6 além dos profissionais muitas vezes terem que lidar com uma organização exigente. A falta de organização no trabalho, pode contribuir para a manifestação de sintomas de sofrimento psíquico, segundo Barbosa et al. (2020).

Outro fator citado nos resultados, foi o da carência de profissional adequado ao manejo de pacientes graves com COVID-19 que pode se dar devido ao profissional não receber treinamentos suficientes para atuar em meio a uma pandemia, fator este que pode resultar em risco ocupacional. A maioria dos profissionais de enfermagem não recebem treinamento o suficiente para lidar em meio a uma pandemia, o que pode resultar em riscos ocupacionais. Esses agravos são ressaltados por 
Da Luz et al., (2020) onde podem causar o Estresse Ocupacional e a Síndrome de Burnout, que também estão evidenciados nos estudos E-1; E-3; E4; E-5; E-7; E-8; E-9; E-11; E-12; que influenciam negativamente na saúde física e mental do enfermeiro (Quadro 2).

Quadro 2 - Identificação dos sinais e sintomas de sofrimento psíquico retratados nos estudos.

\begin{tabular}{|c|l|}
\hline $\begin{array}{c}\text { Principais sinais e sintomas de sofrimento } \\
\text { psíquico }\end{array}$ & \multicolumn{1}{|c|}{ Estudos } \\
\hline \hline Ansiedade & E-1; E-2; E-3; E-4; E-5; E-6; E-7; E-8; E-9; E-10; E-11; E-12 \\
\hline Depressão & E-1; E-3; E-4; E-5; E-6; E-7; E-8; E-9; E-10; E-11; E-12 \\
\hline Insônia & E-1; E-2; E-5; E-8; E-9; E-10; E-11; E-12 \\
\hline Estresse & E-1; E-2; E-4; E-5; E-6; E-8; E-10; E-11; E-12 \\
\hline Estresse pós-traumático & E-1; E-3; E-8; E-12 \\
\hline Medo & E-1; E-4; E-5; E-8; E-9 \\
\hline Esgotamento físico e mental & E-1; E-4; E-6; E-8 \\
\hline Angústia & E-1; E-5; E-8; E-12 \\
\hline Burnout & E-1; E-2; E-3; E-4; E-7; E-8; E-9; E-11; E-12 \\
\hline
\end{tabular}

Fonte: Autores (2021).

Com o objetivo de amenizar o estresse e evitar o adoecimento, os artigos abaixo evidenciaram estratégias de coping que favorecem a atuação do enfermeiro nesse momento delicado, descrito no Quadro 3.

As situações de sofrimento psíquico podem ter se agravado durante a pandemia da COVID-19. Medidas como o acompanhamento psicológico é uma das estratégias mais citadas e favorece a relação interpessoal pois possibilitam ao profissional ressignificar a vida e valores pessoais e assim, desenvolver competências para enfrentamento de adversidades na prática assistencial. Ramos-Toescher et al. (2020) corroboram que em consequência da pandemia da COVID-19 ações psicológicas direcionadas aos profissionais da enfermagem realiza um papel de suma importância para reverter o cenário atual.

Moreira e Lucca (2020) demonstram as ações de capacitação e de apoio social e suporte psicológico são de responsabilidade das instituições de saúde e essenciais para preservação e manutenção da saúde mental que gera melhor condição de continuar com o cuidado ao paciente.

Diante desse cenário, o Conselho Federal de Enfermagem (COFEN) em conjunto com a Comissão Nacional de enfermagem em Saúde Mental disponibilizou como medida de intervenção e apoio o atendimento por meio da teleconsulta de potencial terapêutico direcionados aos profissionais de enfermagem atuantes no período da pandemia da COVID-19 (Humerez; Ohl \& Silva, 2020). 
Quadro 3 - Estratégias de Coping identificada nos estudos.

\begin{tabular}{|c|c|c|c|}
\hline Estudo & Autor & Título do artigo & Estratégia de Coping \\
\hline E-1 & $\begin{array}{l}\text { (MIIRANDA, } \\
\text { et al, 2021). }\end{array}$ & $\begin{array}{l}\text { Sofrimento paiquico entre os profissionais de } \\
\text { enfermagem durante a pandemia da COVID-19: } \\
\text { Scoping }\end{array}$ & $\begin{array}{l}\text { Implementação de ações de capacitação, proteçăo e segurança pelas Instituições de saúde; Suporte e } \\
\text { apoio psicossocial de imediato. }\end{array}$ \\
\hline E-2 & $\begin{array}{l}\text { (DA LUZ et } \\
\text { al., 2020). }\end{array}$ & $\begin{array}{l}\text { Repercussões da covid-19 na saúde mental dos } \\
\text { trabalhadores de enfermagem. }\end{array}$ & $\begin{array}{l}\text { Estratégias para promoção da saúde no trabalho por parte das Instituições governamentais; } \\
\text { Investimentos em acolhimento em saúde mental; Medidas de monitoramento do estresse no trabalho. } \\
\text { Acompanhamento psicológico; Rede de apoio social que visa favorecer os relacionamentos } \\
\text { interpessoais: Criaçấo de diacusgóes coletivas e grupos multiprofisgionais. }\end{array}$ \\
\hline E-3 & $\begin{array}{l}\text { (MOREIRA \& } \\
\text { LUCCA, } \\
2020)\end{array}$ & $\begin{array}{l}\text { Apoio paicossocial e saude mental dos profissionais } \\
\text { de enfermagem no combate ao COVID-19. }\end{array}$ & Apoio social dos colegas de trabalho, chefia e entidades governamentais. \\
\hline E-4 & $\begin{array}{l}\text { (HUMEREZ, } \\
\text { OHL E } \\
\text { SILVA 2020). }\end{array}$ & $\begin{array}{l}\text { Saúde mental dos profissionais de enfermagem no } \\
\text { Brasil no contexto da pandemia de Covid-19: açäo } \\
\text { do Conselho Federal de Enfermagem. }\end{array}$ & $\begin{array}{l}\text { Espaço de intervenção e escuta; considerar as necessidades humanas básicas; ouvir empático, } \\
\text { planejado. }\end{array}$ \\
\hline E-5 & $\begin{array}{l}\text { (BARBOSA et } \\
\text { al., 2020) }\end{array}$ & $\begin{array}{l}\text { Fatores de estresse nos profissionais de enfermagem } \\
\text { no combate a covid-19. }\end{array}$ & $\begin{array}{l}\text { Redução da carga horária de trabalho e/ou aumento do descanso; comunicação; distribuição clara de } \\
\text { tarefas; encaminhar o profissional quando apresentar sinal de estresse ao psicoterapeuta; capacitaçăo } \\
\text { dos profissionaig; incentivo a realizar meditacão e atividades similares para redução do estresse. }\end{array}$ \\
\hline E-6 & $\begin{array}{l}\text { (DAL'BOSCO } \\
\text { et al, 2020) }\end{array}$ & $\begin{array}{l}\text { A gaúde mental da enfermagem no enfrentamento } \\
\text { da COVID-19 em um hospital universitário } \\
\text { regional. }\end{array}$ & $\begin{array}{l}\text { Apoio psicológico; atendimento por telefone que realiza escuta diferenciada, siglosa e gratuita; } \\
\text { realização de práticas integrativas como Yoga, Reiki; realizaçăo de exercícios de relaxamento. }\end{array}$ \\
\hline E-7 & $\begin{array}{l}\text { (SOUZA et } \\
\text { al.,2020). }\end{array}$ & $\begin{array}{l}\text { Trabalho de Enfermagem na pandemia da covidlo e } \\
\text { repercussões para a saúde mental dos trabalhadores. }\end{array}$ & $\begin{array}{l}\text { Promover a comunicação eficiente e eficaz tanto na equipe quanto interpessoal; criar espaço coletivo } \\
\text { para descansar entre os turnos de trabalho; adotar uma boa alimentação; realizar a prática de atividade } \\
\text { fisica; evitar hábitos prejudiciais como o uso de bebidas alcoólicas e outras drogas com o objetivo de } \\
\text { fugir da angústia e tenaão. }\end{array}$ \\
\hline E-8 & $\begin{array}{l}\text { (S.ANTOS et } \\
\text { al. } 2020)\end{array}$ & $\begin{array}{l}\text { Depressão e ansiedade em profisgionais de } \\
\text { enfermagem durante a pandemia da Covid-19. }\end{array}$ & $\begin{array}{l}\text { Realizar a prática de atividades físicas; atendimentos virtuais para suporte aos profisgionais de } \\
\text { enfermagem; eatratégias de promoção da saúde por meio dos órgãos representativos }\end{array}$ \\
\hline E-9 & $\begin{array}{l}\text { (SCHULTZ et } \\
\text { al., 2020). }\end{array}$ & $\begin{array}{l}\text { Resiliência da equipe de enfermagem no âmbito } \\
\text { hospitalar com ênfase na pandemia COVID-19. }\end{array}$ & $\begin{array}{l}\text { Implantação de medidas de prevençăo; medidas de manejo de fatores de risco ergonómicos, que } \\
\text { favorece o menor esforço físico do profissional de enfermagem; prática de atividades físicas; } \\
\text { atividades de lazer; qualificação do cuidado; incentivo a educação contínua; proporcionar serviços de } \\
\text { apoio emocional aos trabalhadores da linha de frente. }\end{array}$ \\
\hline E-10 & $\begin{array}{l}\text { (FERREIRA } \\
\text { et al, 2020). }\end{array}$ & $\begin{array}{l}\text { Uma reflexão sobre saúde mental do enfermeiro } \\
\text { emergencista no contexto da pandemia pelo Covid- } \\
\text { 19. Conselho Federal de Enfermagem. }\end{array}$ & $\begin{array}{l}\text { Incluir as Práticas Integrativas e Complementares (PIC'g) nas rotinas de trabalho como meditação, } \\
\text { auricoloterapia, fitoterapia, musicoterapia, aromaterapia, terapia comunitária integrativa dentre outras }\end{array}$ \\
\hline E-11 & $\begin{array}{l}\text { (RAMOS- } \\
\text { TOESCHER } \\
\text { et al. } 2020)\end{array}$ & $\begin{array}{l}\text { Saúde mental de profissionais de enfermagem } \\
\text { durante a pandemia de COVID-19: recursos de } \\
\text { apoio }\end{array}$ & $\begin{array}{l}\text { Uso da psicoterapia; uso de ações psicoeducativas por meio de cartilhas virtuais, plataformas com } \\
\text { guias informativos, video aulas, e-books; fornecimento de plantôes paicológicos. }\end{array}$ \\
\hline E-12 & $\begin{array}{l}\text { (BEZERRA et } \\
\text { al., 2020). }\end{array}$ & $\begin{array}{l}\text { O impacto da pandemia por COVID-19 na saúde } \\
\text { mental dos profissionais de saúde: revisäo } \\
\text { integrativa. }\end{array}$ & $\begin{array}{l}\text { Ofertar treinamentos adequados; diminuir as jornadas exaustivas de trabalho; meio adequado para } \\
\text { descanso dos profissionais; estratégias de apoio psicológico online. }\end{array}$ \\
\hline
\end{tabular}

Fonte: Autores (2021).

Outras estratégias de coping que são citadas no processo de melhorias na saúde mental do profissional de enfermagem é o aumento do descanso entre as jornadas de trabalho ou a redução da carga horária e a divisão clara de tarefas, pois a sobrecarga e a falta de organização podem gerar sentimento de impotência e sofrimento. Barbosa et al. (2020) asseveram que a flexibilização do horário tem potencial para diminuir o estresse emocional ao qual os profissionais de saúde estão submetidos e a divisão clara de tarefas pode contribuir para o bem-estar, onde permite o enfermeiro se organizar na prestação de cuidados.

A inclusão de estratégias como a prática de atividades físicas é primordial ao homem pois estimula o progresso cardiorrespiratório, osteomuscular e nervoso bem como ameniza a carga psíquica do trabalho. Ao mesmo tempo, a inclusão de uma boa alimentação no dia-a-dia. Souza et al. (2020) asseguram como medidas de redução do sofrimento no trabalho a adoção de uma boa alimentação e incluir a práticas de atividades físicas na rotina.

\section{Considerações Finais}

A pandemia da COVID-19 acarretou um cenário de diversas mudanças na vida das pessoas, principalmente nos profissionais de enfermagem. O presente estudo expõe alguns fatores que influenciam para problemas psíquicos ao qual o profissional de enfermagem se encontra exposto, como a sobrecarga de trabalho, a falta de equipamentos de proteção individual, redução da equipe atuante, a falta de organização no trabalho e condições adequadas para exercer o cuidado ao paciente. Mesmo a enfermagem tendo como o objetivo cuidar de vidas, todo o esforço do profissional pode acabar resultando em danos irreversíveis por não conseguir dar a assistência devida ao paciente pela sobrecarga de trabalho e desencadear o sentimento de frustação e impotência.

Frente a essa situação, se faz necessário implantar medidas de prevenção para minimizar os impactos na saúde mental do enfermeiro atuante na pandemia COVID-19, como ações psicológicas direcionadas aos profissionais da enfermagem para 
ajudar a ressignificar as experiências vividas, implantação de treinamentos adequados por meio das instituições de saúde bem como, a prática de atividades físicas e atividades similares para a redução do estresse, ter o descanso adequado entre as jornadas de trabalho junto a uma boa alimentação, permanecer conectado com a rede socio afetiva a fim de reduzir o sentimento de isolamento, e buscar um profissional adequado quando as estratégias não forem suficientes para a estabilização emocional.

As estratégias de atendimento virtual já acontecem por meio da implementação do COFEN junto a Comissão Nacional de enfermagem em Saúde Mental por meio de atendimento virtual, que está disponível 24 horas por dia. O apoio psicológico e melhores condições de trabalhos são essenciais para exercer o cuidado de qualidade de forma contínua. Os resultados contribuem para a prática assistencial, pois servem de alerta para gestores oportunizarem espaços para discutir e repensar a organização de trabalho.

Para melhor compreensão das repercussões psicológicas na pandemia é necessário entender todo o contexto e as principais implicações e emoções vividas. Logo, mais pesquisas sobre saúde mental, tanto dos profissionais da área da saúde, quanto da população em geral envolvida, precisam ser realizadas pois o conhecimento e a experiencia prévia de situações parecidas podem auxiliar no direcionamento de ações e recursos efetivos.

\section{Referências}

Areosa, J. (2021). Ensaio sobre psicodinâmica do trabalho. Revista Katálysis, 24(2), 321-330. https://doi.org/10.1590/1982-0259.2021.e77288

Barbosa, D. J., et al. (2020). Fatores de estresse nos profissionais de enfermagem no combate à pandemia da COVID-19: Síntese de Evidências. Comunicado em Ciências da Saúde., 31(1), 31-47 https://doi.org/10.51723/ccs.v31iSuppl\%201.651

Bezerra, G. D., et al. (2020). O impacto da pandemia por COVID-19 na saúde mental dos profissionais de saúde: revisão integrativa. Revista de Enfermagem Atual In Derme, 93, e-020012. https://doi.org/10.31011/reaid-2020-v.93-n.0-art.758

Dal'Bosco, E. B., et al. (2020). A saúde mental da enfermagem no enfrentamento da COVID-19 em um hospital universitário regional. Revista Brasileira de Enfermagem, 73(2), e20200434. https://doi.org/10.1590/0034-7167-2020-0434

Da Luz, E. M. F., et al. (2020). Repercussões da Covid-19 na saúde mental dos trabalhadores de enfermagem. Revista de Enfermagem do Centro Oeste Mineiro, 10, e3824. https://doi.org/10.19175/recom.v10i0.3824

Ferreira, F. G. P., et al. (2020). Uma reflexão sobre saúde mental do enfermeiro emergencista no contexto da pandemia pelo Covid-19. Research, Society and Development, 9(7), e704974534-e704974534. https://doi.org/10.33448/rsd-v9i7.4534

Humerez, D. C. de., Ohl, R. I. B., \& da Silva, M. C. N. (2020). Saúde mental dos profissionais de enfermagem do brasil no contexto da pandemia covid-19: ação do conselho federal de enfermagem. Cogitare Enfermagem, 25, e74115. http://dx.doi.org/10.5380/ce.v25i0.74115

Miranda, F. B. G., et al. (2021). Sofrimento psíquico entre os profissionais de enfermagem durante a pandemia da COVID-19: Scoping Review. Escola Anna Nery, 25, e20200363. https://doi.org/10.1590/2177-9465-EAN-2020-0363

Moreira, A. S., \& de Lucca, S. R. (2020). Apoio psicossocial e saúde mental dos profissionais de enfermagem no combate ao COVID-19. Enfermagem em Foco (Brasília), 155-161. ISSN: 2357-707X

Oliveira, V. C., \& de Almeida, R. J. (2017). Aspectos que determinam as doenças osteomusculares em profissionais de enfermagem e seus impactos psicossociais. Journal of Health Sciences, 19(2), 130-135. https://doi.org/10.17921/2447-8938.2017v19n2p130-135

Pereira, M. D., et al. (2020). A pandemia de COVID-19, o isolamento social, consequências na saúde mental e estratégias de enfrentamento: uma revisão integrativa. Research, Society and Development, 9(7), e652974548e652974548. https://doi.org/10.33448/rsd-v9i7.4548

Ramos-Toescher, A. M., et al. (2020). Saúde mental de profissionais de enfermagem durante a pandemia de COVID-19: recursos de apoio. Escola Anna Nery, 24, e20200276. https://doi.org/10.1590/2177-9465-EAN-2020-0276

Rodrigues, C. C. F. M., Santos, V. E. P., \& Sousa, P. (2017). Segurança do paciente e enfermagem: interface com estresse e síndrome de Burnout. Revista Brasileira de Enfermagem, 70(5), 10831088. https://doi.org/10.1590/0034-7167-2016-0194

Santos, K. M. R. D., et al. (2021). Depressão e ansiedade em profissionais de enfermagem durante a pandemia da covid-19. Escola Anna Nery, 25, e20200370. https://doi.org/10.1590/2177-9465-EAN-2020-0370

Soares, M. I., et al. (2016). Saberes gerenciais do enfermeiro no contexto hospitalar. Revista Brasileira de Enfermagem. 69(4), p. 676-683. https://doi.org/10.1590/0034-7167.2016690409i

Souza, M. T. D., Silva, M. D. D., \& Carvalho, R. D. (2010). Revisão integrativa: o que é e como fazer. Einstein (São Paulo), 8(1), 102-106. https://doi.org/10.1590/S1679-45082010RW1134 
Research, Society and Development, v. 10, n. 15, e30101522066, 2021

(CC BY 4.0) | ISSN 2525-3409 | DOI: http://dx.doi.org/10.33448/rsd-v10i15.22066

Souza, N. V. D. D. O., et al. (2021). Trabalho de enfermagem na pandemia da covid-19 e repercussões para a saúde mental dos trabalhadores. Revista Gaúcha de Enfermagem, 42, e20200225. https://doi.org/10.1590/1983-1447.2021.20200225

Schultz, C. C., et al. (2020). Resiliência da equipe de enfermagem no âmbito hospitalar com ênfase na pandemia COVID-19. Research, Society and Development, 9(11), e539119466-e539119466. https://doi.org/10.33448/rsd-v9i11.9466 